Ciencia y Salud, Vol. III, No. 2, mayo-agosto, 2019 •ISSN (impreso): 2613-8816 • ISSN (en línea): 2613-8824

DOI: https://doi.org/10.22206/cysa.2019.v3i2.pp49-54

\title{
ANÁLISIS COMPARATIVO DEL CONOCIMIENTO DE RCP EN LEGOS Y PERSONAL MÉDICO DE UNA UNIVERSIDAD Y UN HOSPITAL DE SANTO DOMINGO, REPÚBLICA DOMINICANA, DURANTE EL PERÍODO FEBRERO-ABRIL 2015
}

\section{Comparative Analysis of the Knowledge of CPR in Layman and Medical Staff of a University and Hospital of Santo Domingo, Dominican Republic, during the period February-April 2015}

\author{
Pablo Smester ${ }^{1 *}$, Alicia Bueno Fermín ${ }^{2 * *}$, Alan Lanurias Díaz ${ }^{2 * * *}$, Alejandra Rodríguez \\ Heinsen ${ }^{2 * * * *}$, Emely Vásquez Sánchez ${ }^{2 \Delta}$, Scarllen Pérez ${ }^{2 \Omega}$, Lisely Tavárez ${ }^{2 \nvdash}$
}

Recibido: enero 16, 2019 • Aprobado: marzo 16, 2019

Cómo citar: Smester P, Bueno Fermín A, Lanurias Díaz A, Rodríguez Heinsen A, Vásquez Sánchez E, Pérez S, Tavarez L. Análisis comparativo del conocimiento de RCP en legos y personal médico de una universidad y un hospital de Santo Domingo, República Dominicana, durante el período febrero-abril 2015. cysa [Internet]. 26 de julio de 2019 [citado 26 de julio de 2019];3(2): 49-54. Disponible en: https://revistas.intec.edu.do/index.php/cisa/article/view/1478

\section{Resumen}

Introducción: el nivel de conocimiento en legos y personal médico incide en la calidad y eficacia de la Reanimación Cardiopulmonar (RCP).

Objetivo: comparar el nivel de conocimiento y técnicas de RCP en legos y personal médico de una universidad y un hospital de Santo Domingo, República Dominicana, durante el período febrero-abril 2015.

Material y métodos: fue un estudio prospectivo, experimental y de corte longitudinal en el que se evaluaron 30 legos de una universidad de Santo Domingo y 17 residentes de emergenciología de un hospital de la misma ciudad.

Resultados: sin ninguna intervención 12 de 30 legos evaluados (40\%), tuvieron dominio del conocimiento básico de RCP. 12 de 17 (70.58 \%), tuvieron un conocimiento

1. Profesor y director del Laboratorio de Simulación, Instituto Tecnológico de Santo Domingo (INTEC)

2. Estudiantes del INTEC

Abstract

Introduction: The level of knowledge in laymen and medical personnel affects the quality and efficacy of Cardiopulmonary Resuscitation (CPR).

Objective: Is to compare the level of knowledge and techniques of CPR in laymen and medical personnel of a university and a hospital in Santo Domingo, Dominican Republic, during the period February-April 2015.

Material and Methods: This was a prospective, experimental and longitudinal cutting study in which 30 laymen from a university in Santo Domingo and 17 emergency residents from a hospital were evaluated.

Results: Without intervention, 12 of 30 laypersons (40\%) had basic knowledge of CPR. 12 of 17 (70.58\%) had a basic

Correo-e de los autores:

* pablo.smester@intec.edu.do

** ferminaliciab@gmail.com

***alan_lanuriasdiaz@hotmail.com
**** alecristi06@hotmail.com

$\Delta$ Emely.vasquez01@gmail.com $\Omega$ scarllen.perez@intec.edu.do $\forall$ li2025_08@hotmail.com 
básico de RCP. Por otra parte, tanto el $100 \%$ de los residentes como de los legos presentaron un conocimiento acertado de RCP luego de la intervención.

Conclusiones: el nivel de conocimiento en una población estudiantil antes de una intervención presentó valores menores en relación con un personal médico ya entrenado. Sin embargo, estos valores sufrieron un cambio luego de la intervención realizada a la población estudiantil provocando un aumento de los valores en la comparación con legos y personal médico.

Palabras clave: reanimación cardiopulmonar; lego; paro cardíaco; paro respiratorio; personal médico.

\section{Introducción}

A pesar de la existencia de información acerca de la RCP (Reanimación Cardio Pulmonar), existe un gran déficit de conocimiento en torno a la práctica. Por tal motivo, es de gran interés que a través de investigaciones como esta quede demostrada la importancia del conocimiento de la técnica de RCP "solo manos". Estudios recientes muestran que los paros cardíacos y paros respiratorios son las principales causas de muerte en República Dominicana ${ }^{1}$. Según expertos del Hospital General Plaza de la Salud (HGPS) y el Departamento de Educación Médica Continua (DEMEC) de la Universidad Iberoamericana (UNIBE), la RCP temprana y adecuada salva vidas. Se ha determinado a su vez, en las investigaciones citadas, que las víctimas por paros cardiorrespiratorios tienen más probabilidades de sobrevivir cuando un RCP efectivo se aplica inmediatamente ${ }^{2}$.

La reanimación cardiopulmonar $(\mathrm{RCP})$ consiste un conjunto de técnicas organizadas que se utilizan para poner en correcta función la actividad cardiopulmonar ${ }^{4}$. En la mayoría de los casos se utiliza la técnica de RCP tras un paro cardiorrespiratorio (PCR). Siendo esta la situación clínica que cursa con interrupción brusca, inesperada y potencialmente reversible de la actividad mecánica del corazón y de la respiración espontánea ${ }^{5}$. understanding of CPR. On the other hand, both $100 \%$ of the residents and the laymen, presented an accurate knowledge of CPR after the intervention.

Conclusions: The level of knowledge in a student population before an intervention presented lower values in relation to a medical staff already trained. However, these values underwent a change after the intervention made to the student population causing an increase in the values in comparison with laymen and medical personnel.

Keywords: Cardiopulmonary resuscitation; layman; cardiac arrest; Respiratory Arrest; medical staff.

El propósito de la reanimación cardiopulmonar es mantener la ventilación pulmonar y la circulación sanguínea suficientes, para mantener el aporte de oxígeno al cerebro hasta que puedan obtenerse los medios que puedan revertir la causa subyacente de la parada ${ }^{8}$.

Para aplicar RCP se deben tener en cuenta los siguientes pasos, basados en las instrucciones de la American Heart Association ${ }^{9,10}$.

1. Verificar si hay respuesta de la persona.

2. Llamar al número local de emergencias, si no hay respuesta.

3. Colocar a la persona cuidadosamente boca arriba. Si existe la posibilidad de que la persona tenga una lesión en la columna, dos personas deben moverla para evitar torcerle la cabeza y el cuello.

4. Realizar compresiones cardíacas o pectorales: aplique 100 compresiones por minuto para restaurar el sistema cardiopulmonar.

5. Abrir la vía respiratoria. Levantarle la barbilla con dos dedos. Al mismo tiempo, inclínele la cabeza hacia atrás empujando la frente hacia abajo con la otra mano. 
6. Observe, escuche y sienta si hay respiración.

7. Si la persona no está respirando o tiene dificultad para respirar: dé dos insuflaciones boca a boca.

8. Continúe la RCP hasta que la persona se recupere o llegue ayuda.

\section{Materiales}

Esta investigación, de acuerdo al tiempo, fue de carácter prospectivo y bibliográfico. De igual modo, de corte longitudinal; se tomó como estudio experimental ya que se intervino sobre la variable independiente, observando si hubo cambios o no en los sujetos de estudio. Por último, fue una investigación de campo, ya que se ejecutó en el lugar del estudio, conociendo las características del mismo.

Se finalizó el estudio con 47 personas, siendo estas 30 estudiantes de una universidad y 17 residentes del área de emergencias de un hospital de Santo Domingo, República Dominicana, correspondientes tanto a la población como a la muestra.

La investigación conllevó los siguientes criterios de inclusión: personas que desearon participar en el estudio, personas que completaron todas las fases, estudiantes activos de una universidad y residentes del área de emergencia de un hospital. Todos los participantes $(100 \%)$ firmaron un consentimiento informado.

Como instrumento de recolección de datos se utilizó un formulario elaborado por los autores en conjunto con el asesor, el cual se empleó para medir los conocimientos, actitudes y prácticas tanto del personal lego como del personal médico, inmediatamente antes y después del entrenamiento en RCP "solo manos". Un mes más tarde se evaluaron nuevamente los conocimientos y actitudes por medio de otro formulario redactado por el asesor y los autores.

\section{Resultados}

Gráfica 1. Nivel de conocimiento de RCP antes de la intervención. $(\mathrm{N}=47)$

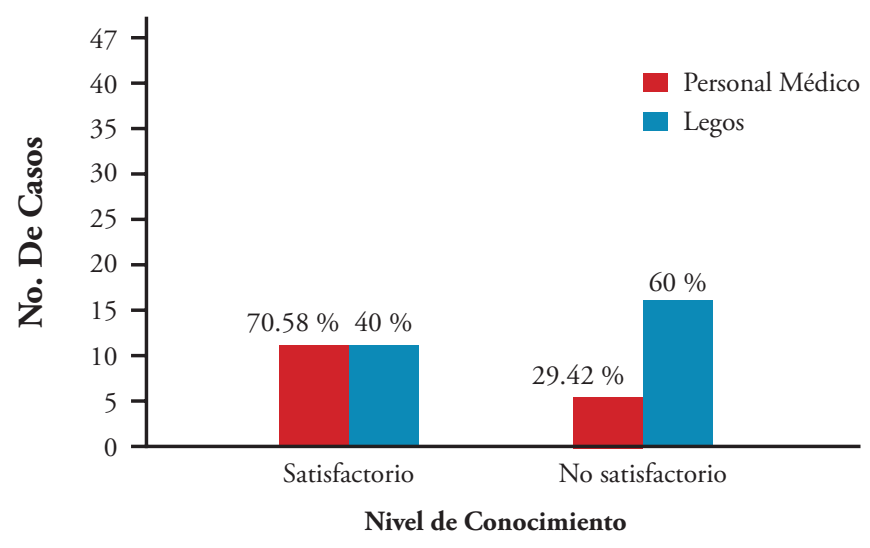

Fuente: BioINTEC Jornada 68.

En el primer grupo, 12 de 17 residentes del área de emergencia del hospital seleccionado mostraron un dominio del conocimiento de RCP, representando un $70.58 \%$. El $29.42 \%$ restante, es decir, 5 residentes, no tenía técnicas y conocimientos de RCP antes de la intervención. Luego, al evaluar los legos se obtuvo un resultado totalmente distinto, ya que 12 legos de 30, esto es, el $40 \%$, respondieron correctamente las evaluaciones, mientras que el $60 \%$ restante, representando 18 legos, no poseía conocimiento de RCP.

Gráfica 2. Nivel de conocimiento de RCP después de la intervención. $(\mathrm{N}=47)$

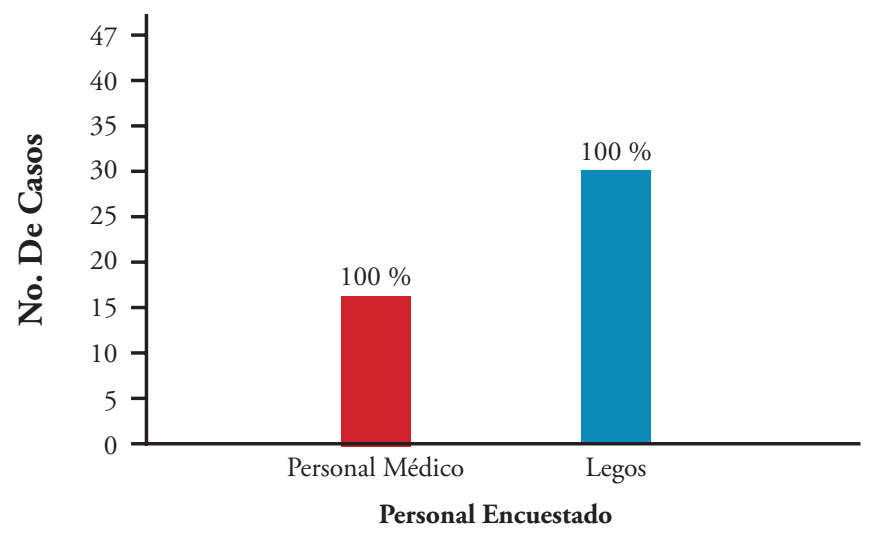

Fuente: BioINTEC Jornada 68. 
En el primer grupo, todo el personal médico, es decir, 17, mostraron dominio del conocimiento de RCP, representando un $100 \%$. De igual forma, los legos obtuvieron el $100 \%$, 30 tuvieron las evaluaciones correctamente respondidas.

Gráfica 3. Nivel de efectividad de las técnicas RCP "solo manos" durante la intervención. ( $\mathrm{N}=47)$

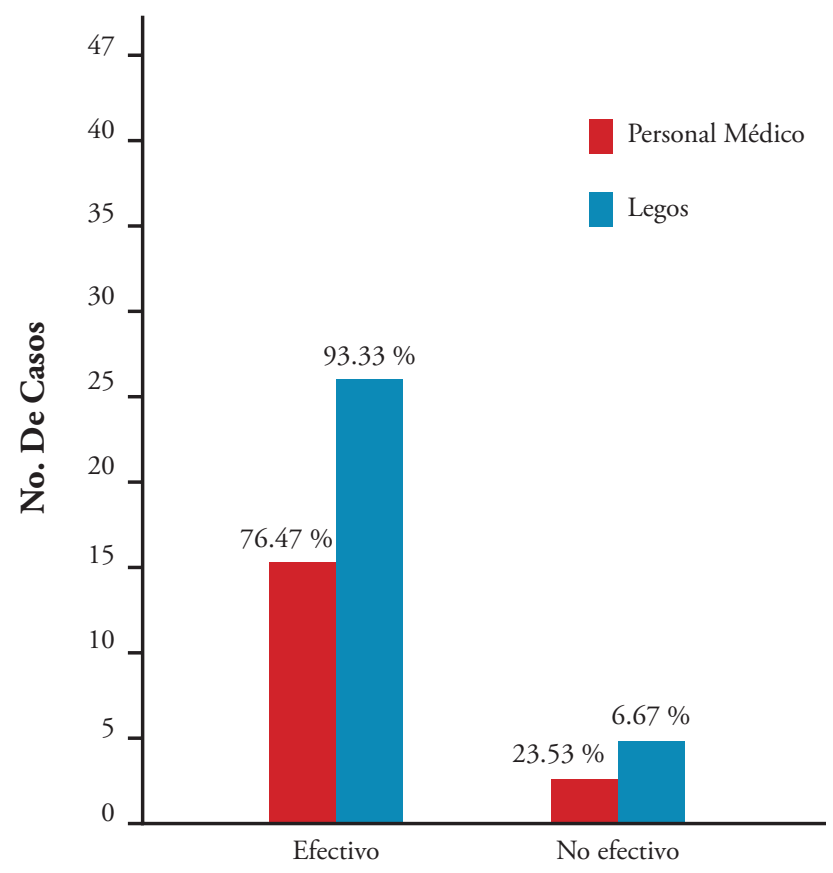

Nivel de Conocimiento

Fuente: BioINTEC Jornada 68.

En el primer grupo, 13 representantes del personal médico mostraron una correcta aplicación del RCP "Efectivo" durante la intervención, es decir, el $76.47 \%$, siendo el $23.53 \%$ restante "No efectivo", lo que nos dices que 4 integrantes del personal médico no aplicaron correctamente las técnicas del $\mathrm{RCP}$ con el maniquí durante la intervención.

En el segundo grupo, los legos obtuvieron el 93.33\% "Efectivo", es decir, 28 legos mostraron una correcta aplicación del RCP durante la intervención, mientras que el $6.67 \%$ restante "No efectivo", no aplicó correctamente las técnicas de RCP.
Gráfica 4. Nivel de conocimiento de la información trasmitida durante el entrenamiento después de un mes. $(\mathrm{N}=47)$

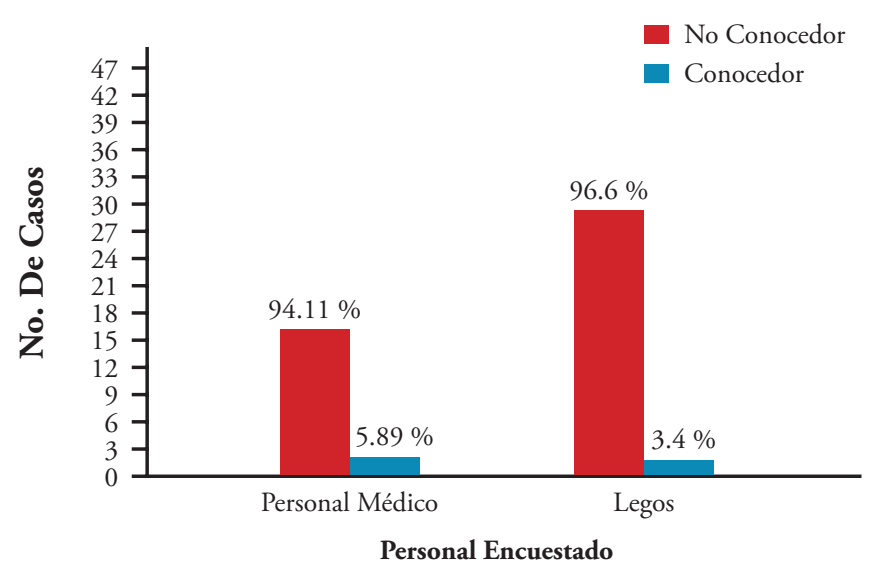

Fuente: BioINTEC Jornada 68.

En el primer grupo, 16 integrantes del personal médico mostraron dominio de la información transmitida, es decir, el $94.11 \%$. El $5.89 \%$ restante, es decir, 1 integrante del grupo personal médico no respondió correctamente la evaluación sobre RCP.

En el segundo grupo, los legos obtuvieron el 96.6\%, es decir, 29 legos mostraron dominio de la información transmitida durante el entrenamiento, sin embargo, el $3.4 \%$ restante, es decir, 1 integrante del grupo lego, no respondió correctamente la evaluación.

\section{Discusión y conclusiones}

Los doctores Alarcón Peralta, Christian Miguel et al., (2010) en asesoría con el Dr. William Francisco Medina Chamaidan, realizaron un estudio titulado "Evaluación de conocimientos en Reanimación Cardiopulmonar en el personal médico del área de emergencia en principales hospitales de Guayaquil". Para ello fueron encuestados 115 (51.3\%) estudiantes internos en medicina y 109 (48.7\%) médicos residentes ${ }^{12}$. De estos, un $10 \%$ obtuvo una nota menor a 40, concluyendo así que el personal médico en el área de emergencia, en dichas unidades hospitalarias, poseía deficiencias graves sobre 
los conocimientos de RCP básico y avanzado ${ }^{12}$. Lo cual, de parte del grupo lego, refleja lo obtenido en esta investigación, debido a que solo el $40 \%$ de los legos antes de la intervención mostraron dominio del conocimiento del RCP. Con respecto al grupo del personal médico, estos no presentaron ninguna deficiencia grave sobre los conocimientos de RCP.

Hasta ahora, no se ha encontrado ningún estudio que muestre la relación de un grupo lego y un grupo de personal médico después de una intervención; sin embargo, en esta investigación, tanto el grupo lego y el grupo del personal médico mostraron la misma eficacia, siendo el $100 \%$ en ambos. Por lo cual, da a entender que una intervención promueve los mismos resultados en un grupo no capacitado y en un grupo previamente capacitado.

En conclusión, quedó demostrado que el nivel de conocimiento de RCP en una población estudiantil antes de una intervención crítica presentó valores menores en relación con un personal médico ya entrenado y capacitado. Estos datos obtenidos se apoyaron de los formularios entregados a los grupos de estudio durante la Jornada de RCP impartida en una universidad, el día 26 de febrero del año 2015 y durante una visita a un hospital de la ciudad de Santo Domingo, República Dominicana. Sin embargo, los mismos sufrieron un cambio luego de la intervención realizada a la población estudiantil, provocando un aumento de los valores en comparación con los legos. Con estos datos se verificó que un grupo lego puede poseer una capacitación igual o mayor a la de los residentes médicos del área de emergencia.

Luego de la realización de este estudio se recomienda:

La realización de campañas de información, educación y comunicación a nivel nacional acerca de la Reanimación Cardiopulmonar, y de cómo manejarse ante una situación que requiera de esta técnica. También incentivar a los encargados de las universidades de la República Dominicana para que lleven a cabo una red de charlas del RCP y se logre, de tal manera, transmitir la importancia de las técnicas y conocimientos de RCP. Por último, realizar más trabajos de investigación de esta misma índole, para así tener un mayor registro a nivel nacional sobre el conocimiento del RCP.

\section{Bibliografía}

1. Desde la República Dominicana. Estadísticas de mortalidad en República Dominicana. [Online] Available from: http://desdelarepublicadominicana.blogspot.com/2010/09/ estadisticas-de-mortalidad-en-republica.html [Accessed $1^{\text {st }}$ September 2010].

2. Victor Navarro $M$, Gabriel Rodríguez $S$. Reanimación Cardiopulmonar Básica. SCLD. [Online]. 2010. [Consultada 20 de febrero 2015];33(16): 5-8 Available from: http://www. sld.cu/galerias/pdf/sitios/urgencia/4rcp.pdf.

3. Barreto Caballero L. (2011) Nivel de conocimiento del personaje de enfermería sobre las Guias de Resucitación Cardiopulmonar (RCP) de la Asociación Americana del Corazón (AHA) en arresto cardiorrespiratorio en el adulto (tesis doctoral). Centro Universitario de Bayamón, Bayamón, Puerto Rico.

4. Leyda Novel P, Lopez JG, Domínguez de La Llera G. Paro cardiorrespiratorio (PCR). Etiología. Diagnóstico. Tratamiento. Rev Cubana Cir [Internet] 2006 [consultada 10 de marzo 2015];45(3-4). Available from: http://www.bvs. sld.cu/revistas/cir/vol45_3_06/cir19306.htm.

5. Carpio Guzmán R, López Amanzo C, Bautista Bendezu J, Chávez Álvarez C, Tapia Risco E, Garcia Correa RI, Palomino Márquez M. Reanimación Cardio Pulmonar Básico: Conceptos Generales. En: Bolívar Murgueytio L, Dir. Escuela de Emergencias. Guía de Reanimación Cardio Pulmonar Básica. Perú; 2011. p. 9-11. Available from: http://www. essalud.gob.pe/downloads/escuela_emergencia/ GUIA_CARDIOPULMONAR.pdf. 
6. Paro Cardiorespiratorio. Universidad Católica de Chile. [Online]. Available from:http://escuela.med. puc.cl/publ/Aparatorespiratorio/56ParoCardio. html [Accessed 20th February 2015].

7. Martínez B. Drogas en RCP extrahospitalaria. Enferurg. [Online]. Available from: http:// www.enferurg.com/articulos/drogasrcp.htm [Accessed 20th February 2015].

8. RCP Adulto y Lactante. Cruz de la vida. Weblog. [Online]. Available from: http://cruzdelavida-lauris. blogspot.com/p/rcp-adulto-y-lactante.html [Accessed 20th February 2015].

9. Chameides L, Hemphill R, Samson R, Sinz E. CPR Guidelines. American Heart Association (AHA). [Online] 2011[consultada 27 de febrero 2015] 73(50): p: 3-28. Available from: https:// www.heart.org/idc/groups/heart-public/@wcm/@ ecc/documents/downloadable/ucm_317350.pdf.

10. European Resuscitation Council Guidelines for Resuscitation. European Resuscitation Council. [en línea] 2010 [consultada 27 febrero 2015]. Available from: https://www.erc.edu/index.php/ docLibrary/ru/viewDoc/1195/3/.

11. Declaración de Helsinki de la AMM - Principios éticos para las investigaciones médicas en seres humanos. World Medical Association.2013. [Online]. Available from: http://www.wma.net/ es/30publications/10policies/b3/. [Accessed 20th February 2015].

12. Alarcón M, Chang E, Ena M, Vinuesa B, Vinko $\mathrm{H}$ et al. Evaluación de conocimientos en Reanimación Cardiopulmonar en el personal médico del área de emergencia en principales hospitales de Guayaquil. MedPre [Internet]. Septiembre 2013 [consultada 20 de marzo 2015];3(1): 44-52. Available from: http://www. medpre.med.ec/ojs/index.php/revistamedpre/ article/download/78/76.
13. Farto Ramírez O, Barcala Furelos R, AbeleirasGómez C. Conocimiento, Ejecución Y Percepción Sobrela RCPen Socorristas. Grupo de Investigación en Rendimiento y Motricidad del Salvamento y Socorrismo (REMOSS). Universidad de Vigo. Facultad de CC. De la Educación y del Deporte. [Online]. Available from: https://docplayer. es/43610167-Conocimiento-ejecucion-y-percepcion-sobre-la-rcp-en-socorristas.html [Accessed $20^{\text {th }}$ February 2015].

14. Ramírez Caballero, R. (2013) Conocimientos y actitudes sobre reanimación cardiopulmonar (RCP) en residentes de Pediatría del Hospital Roosevelt durante noviembre de 2012 (tesis de grado Universidad Rafael Landívar) Recuperado de: http://biblio3.url.edu.gt/Tesario/2013/09/03/ Ramirez-Rodolfo.pdf.

15. Rojas L, Aizman A, Arab J, Utili F, Andersen M. Reanimación cardiopulmonar básica: conocimiento teórico, desempeño práctico y efectividad de las maniobras en médicos generales; Revista Médica de Chile [Internet]. 2012;140: 73-7. Available from: http://www.scielo.cl/pdf/ rmc/v140n1/art10.pdf [Accessed 20th February 2015]. 